\title{
Safety and efficacy demonstrated for novel bipolar radiofrequency ablation system
}

Successful use of a radiofrequency ablation (RFA) device that utilizes two electrodes rather than one has been demonstrated in a small cohort of patients with kidney cancer. The study, reported by Louis Kavoussi and colleagues from New York, was recently published in BJU International.

Standard RFA equipment is monopolar, and associated with a number of limitations - single electrodes are thought to be the cause of collateral damage, lesions of inconsistent size, and asymmetric borders-owing to the cooling effect of nearby blood vessels.

\section{4 ...thermal energy is isolated between the two [electrodes] while adjacent tissue remains untouched... 77}

The newly developed bipolar RFA system consists of two electrodes-an inner straight electrode and an outer corkscrew-shaped electrode-which ensures that thermal energy is isolated between the two while adjacent tissue remains untouched. Preliminary studies in animal models and ex vivo kidney tissue have demonstrated consistent and precise ablation with this system.

Kavoussi and his team evaluated the device in patients undergoing laparoscopic partial or radical nephrectomy for renal tumors $(n=10)$. Ablated specimens were analyzed by a single pathologist, who performed nicotinamide adenine dinucleotide staining and recorded lesion size and shape, as well as width of the transition zone to viable tissue.

The procedure was successful in all 10 patients, with no viable cells detected in ablated tissue. Defined, reproducible lesions were achieved with a mean size of $6.26 \mathrm{~cm}^{2}$ and regular borders. The transition zone ranged from $10 \mu \mathrm{m}$ to
$60 \mu \mathrm{m}$, indicative of a precise procedure. No complications, which might include skin burns and collateral damage, were reported. The authors conclude that further follow-up studies are required to determine the oncological efficiency of this bipolar system.

Ablative surgery has revolutionized the management of small renal masses, and RFA is now an accepted alternative to open nephrectomy. Given the recent data demonstrating that RFA has longterm oncological outcomes comparable to partial nephrectomy, it seems likely that we will see more attempts to refine the RFA procedure in the future.

Sarah Payton
Original article Okhunov, Z. et al. Clinical evaluation of a novel bipolar radiofrequency ablation system for renal masses. BJU Int. doi.10.1111/j.1464410X.2012.10940.x 